\title{
eScience in the Cloud: A MODIS Satellite Data Reprojection and Reduction Pipeline in the Windows Azure Platform
}

\author{
Jie Li*, Deb Agarwal**, Marty Humphrey*, Catharine van Ingen***, Keith Jackson**, and \\ Youngryel Ryu**** \\ * Department of Computer Science, University of Virginia, Charlottesville, VA USA \\ ** Lawrence Berkeley National Lab, Berkeley, CA USA \\ *** Microsoft Research, Microsoft Bay Area Research Center, San Francisco, CA USA \\ ****Dept. Environmental Science, Policy and Management, UC-Berkeley, Berkeley, CA USA
}

\begin{abstract}
The combination of low-cost sensors, low-cost commodity computing, and the Internet is enabling a new era of data-intensive science. The dramatic increase in this data availability has created a new challenge for scientists: how to process the data. Scientists today are envisioning scientific computations on large scale data but are having difficulty designing software architectures to accommodate the large volume of the often heterogeneous and inconsistent data. In this paper, we introduce a particular instance of this challenge, and present our design and implementation of a MODIS satellite data reprojection and reduction pipeline in the Windows Azure cloud computing platform. This cloud-based pipeline is designed with a goal of hiding data complexities and subsequent data processing and transformation from end users. This pipeline is highly flexible and extensible to accommodate different science data processing tasks, and can be dynamically scaled to fulfill scientists' various computational requirements in a costefficient way. Experiments show that by running a practical large-scale science data processing task in the pipeline with 150 moderately-sized Azure virtual machine instances, we were able to produce analytical results in nearly $90 \mathrm{X}$ less time than was possible with a high-end desktop machine. To our knowledge, this is one of the first eScience applications to use the Windows Azure platform.
\end{abstract}

\section{Introduction}

The combination of low-cost sensors, low-cost commodity computing, and the Internet is enabling a new era of data-intensive science. Many scientists are envisioning analysis and synthesis computations that can easily go beyond tera-scale or even peta-scale data capabilities. For example, remote sensing is one of the factors transforming environmental science today. Emerging inexpensive ground-based sensors are enabling scientists to make field measurements of science variables at more locations with more spatial and temporal granularity. Satellites and other remote sensing technologies can at times provide a second source of measurements to the data generated by the ground sensors, and at other times serve as the single source of data for those parts of the Earth that are not amenable to ground-based sensors. Large-scale environmental data is necessary to investigate global-scale phenomena such as global warming.

Given these remarkable technological improvements, scientists can increasingly find the sensor data they need on the Internet, or they can design and deploy a custom software 
architecture to generate the sensor data they need. But now scientists are facing with a new challenge: how to process the data. First, the source data from different locations or measurements can be very heterogeneous in data format, resolution, time frame, confidence, etc., making it difficult for scientists to use directly in their research. Second, deriving from this first issue, the best algorithms can often not be determined until the raw data is actually available, because the algorithms designed under ideal situations often do not account for the less-thanideal raw data. Third, the inherent nature of the scientific hypotheses and experiments often require a scale beyond which the scientists have previously encountered. A scientist's commodity workstation will quickly be overwhelmed for non-trivial computations over large datasets. Purchasing larger computational platforms (i.e., clusters) - although arguably the norm - is generally not cost-effective even if the money is available, as they quickly become outdated and especially if additional system administrators must be hired by the domain scientists just to manage the infrastructure. Obtaining an account and allocation on national-scale supercomputing centers can be pursued but frequently offers a particular programming and debugging environment that is not as flexible as a local option, and user jobs can sometimes require a potentially long wait in a queuing system.

This paper is motivated by a particular instance of this problem, as we attempted to integrate data from ground-based sensors with the Moderate Resolution Imaging Spectroradiometer (MODIS) [1][2] data. Designed to improve the understanding of global dynamics and processes occurring on the land, oceans, and lower atmosphere, the MODIS data is generated by the Terra and Aqua satellites and is a viewing of the entire Earth's surface in 36 spectral bands, at multiple spatial resolutions, generated every 1-2 days. There are a large number of research activities that are using the MODIS data to explore and validate scientific hypotheses (e.g., see [3] for an overview with regard to vegetation and [4] for an overview with regard to ocean science). We encountered all three issues identified above, particularly the inability to practically compute such data transformation and integration on a scientist's desktop machine. As for our example, even a high-end desktop machine with Quad-core processing would require tens of months of continuous processing to compute our result (details are provided later in this paper). Furthermore, our desire to expose this as a Web service for the broader environmental research community could not possibly be implemented if we used this high-end desktop machine.

In this paper, we describe our experiences designing and implementing our MODIS data integration and transformation techniques by using cloud computing, specifically the Windows Azure platform [5]. To our knowledge, this is one of the first science applications to use the Windows Azure platform. The contributions of this paper are:

- We present a novel approach to "reproject" the input data into timeframe- and resolutionaligned, and uniform geographically formatted data.

- We present a novel "reduction" technique to derive important new environmental data through the integration of satellite and ground-based data.

- We describe how we leveraged the Windows Azure abstractions and APIs to accomplish the reprojection and reduction steps.

- We present our general observations and lessons learned as we explore how emerging common cyberinfrastructure such as Windows Azure can be leveraged by resourceconstrained domain scientists.

While cloud computing is still in its infancy and many challenging open issues remain, we believe that the Windows Azure platform is a compelling approach for large-scale scientific explorations, as, for example, we gained a factor of 90x speedup over a high-end desktop 
machine when running 150 Azure virtual machine instances for parallel data reprojection tasks. Although the project was developed specifically for MODIS data processing, we believe that the experience we gained and lessons learned from implementing this architecture in Windows Azure can be applied to a broad range of other imaging eScience applications.

The remainder of this paper is structured as follows. Section 2 discusses existing use of cloud computing for eScience. Section 3 gives an overview of Windows Azure. In section 4 we introduce the background of our project. Section 5 introduces the implementation details of our pipeline architecture. Section 6 shows the evaluation results of the pipeline. In section 7 we conclude our work with discussions on our experience with Windows Azure and some general observations and unresolved issues about leveraging cloud computing for eScience.

\section{Existing Use of Cloud Computing for eScience}

Commercial cloud computing platforms such as Amazon's Elastic Compute Cloud (EC2 [6]), Google App Engine [7], and Microsoft's Windows Azure have been created to offer highly flexible, scalable, and on-demand computational, storage, and networking resources for largescale computing tasks. It can be argued that the emphasis of these commercial clouds is at least perceived to be for "business applications", so it is not clear how readily such commercial clouds can be used for science. We believe such platforms have the potential to be a cost-efficient computing platform for domain scientists, particularly due to the economies of scale and increasing market competition, which will drive the costs down. Furthermore, the pay-as-you-go manner in the business model of cloud computing means scientists no longer need any specific authorization for using powerful computing resources and there is no large up-front cost.

The number of Cloud science applications has been increasing. Evangelinos and Hill pursue the use of EC2 for Atmospheric-Ocean models [8]. The CARMEN e-science project is designing a system to allow neuroscientists to share, integrate and analyze data, and has been recently expanded to include cloud computing [9]. Keahey et. al. describe early experiences to deliver EC2-like cycles to scientific applications [10]. The feasibility of executing workflows in the cloud is being studied, particularly for the Montage application [11]. (Montage is the subject of another cloud-based analysis in [12]). Analysis of gene expression data and microarrays is being pursued in public clouds [13]. Other studies are beginning to appear that address the performance of science applications in the cloud [14][15]. While these efforts and others are pioneering the use of science applications in the cloud, we believe that there are many unresolved issues on how, in particular, domain scientists can best leverage and exploit cloud computing. We believe our cloud-based environmental data analysis reported in this paper complements this existing research by reporting the first experiences using Windows Azure for escience.

\section{Windows Azure overview}

Windows Azure was announced by Microsoft as its cloud computing service platform in Microsoft Professional Developers Conference (PDC) 2008. Early access in the Community Technical Preview phase occurred in spring 2009. Commercial availability is expected to be announced in November 2009 at the same time as PDC 2009.

Windows Azure presents a .NET-based hosting platform that is integrated into a virtual machine abstraction. Thus, developers who are familiar with .NET application development can take advantage of this homogeneous cloud environment and develop applications for Azure just like ordinary .NET applications by using Visual Studio. In contrast to EC2, Windows Azure does 
not expose the virtual machine abstraction directly to an end-user. In EC2, users can customize the environment for their particular application by installing particular software or by purchasing particular machine images. Windows Azure achieves flexibility via the wide range of languages supported by Visual Studio and the .NET framework, and Windows Azure support popular standards and protocols including SOAP, REST, XML, and PHP.

In Windows Azure, the virtual machine instances can be separated into two different roles: the front-end website hosting server instances are called Web Roles, and the back-end computational instances are called Worker Roles. Developers can specify the number of instances for both roles at the deployment of their application, or (in the near future) can dynamically adjust the number of instances at runtime.

Besides the computational resources provision, Windows Azure also provides three types of cloud storage services:

- Blob service, the main storage service for storing durable large data items;

- Queue service, which provides a basic reliable queue model to allow asynchronous task dispatch and to enable service communication;

- Table service, which provides the structured storage in the form of tables and supports simple queries on partitions, row keys, and attributes.

The key aspect of cloud storage is that it is accessible via any virtual machine in Azure (with the proper authentication/authorization). Therefore, while there is local storage available to a particular computation, it is assumed that one of the cloud storage services will be used if the data is to be shared across virtual machine instances.

\section{MODIS Satellite Data Processing}

As mentioned earlier, the MODIS data is generated by the Terra and Aqua satellites and is a viewing of the entire Earth's surface in 36 spectral bands, at multiple spatial resolutions, generated every 1-2 days. MODIS provides various biophysical variables (e.g. gross carbon uptake, albedo etc) with spectral irradiance ranging visible, near infrared, infrared and thermal regions of the electromagnetic spectrum. It has been an important scientific source and can be applied to many environmental studies from local to global scale. The MODIS data is made available over the Internet and is on multiple FTP sites every day.

There are three main barriers for scientists to obtain the L2/L3 MODIS data and apply analyses on them to produce scientific results in their research:

Data Collection: The L2/L3 data is separated into 3 main groups: Atmosphere, Land, and Ocean. Each data group includes a number of different data products, which are published and maintained on different FTP sites. For each day the L2/L3 data are stored in tens to hundreds separate pieces of files and the corresponding metadata such as earth covering boundaries, observing time, etc. are kept in a different place. Although there is a web portal [16] for data download by queries (geographic area, time period, data products, etc.), it only supports queries for a very limited data size (no more than $2 \mathrm{~GB}$ ). If a scientist wants to download a larger size of the L2/L3 data, he/she needs to download them directly from the FTP sites, which is almost infeasible given the data size is large and no tools are available for metadata query in order to decide which set of data files to download.

Data Transformation: Data products from different groups are stored in different geographical project types. For example, atmosphere data products are directly derived from the MODIS instrument and use a swath space and time system that follows the satellite, while the 
land data products have already been preprocessed and use a gridded space and time coordinates called the sinusoidal tiling system. The reprojection from one type to the other involves very complex data processing algorithms, and requires in-depth programming skills which are not common to the domain scientists. A number of tools [17][18] have been developed by the community to perform the reprojections. However, these tools only work for a single data file input and lack support for processing/producing an on-demand area and time period of data. Thus, these tools are of limited value when scientists need to synthesis a large space and time scope of data. Furthermore, data from different products can have different time frames and space resolutions, which also need to be unified before scientists could conduct scientific computation on them. This data heterogeneity makes it extremely difficult for scientists to use data from different products directly in their analytical processes, and a main data transformation step is required to produce timeframe- and resolution-aligned, and uniform geographically projected data.

Data Management: To date, most scientific research involving the MODIS data are limited to a small geographical area. It is not feasible to conduct scientific research in continental or global scope not only because of the above two barriers, but also because the data management cost would become overwhelming. For example, the research reported in this paper is motivated in part by a group of biometeorologists needing to conduct a MODIS data synthesis involving nine data products covering the whole US continent in a 10 year time frame. Table 1 contains a description of the nine data products, including the total size of the L2/L3 data the scientists will have to manage. \#Source Data Files indicates the total number of source data files that need to be downloaded for each data product for the whole US over 10 years, and Data Size indicates the size of each data product that needs to be downloaded from the multiple FTP sites. Prior to the research reported in this paper using Windows Azure, these scientists would first need to query the metadata to decide the subset of source data files which covers the US area, download all the source data files to their local storage, reproject the 5 Swath type products into Sinusoidal type, unify the timeframe and space resolution of all 9 data products, and finally perform scientific computation on the data. This process would need to be managed and performed manually by these scientists. This process as a whole is very time-consuming, tedious and error-prone.

Table 1. Example Data Product Requirements for US Continent over 10 Years

\begin{tabular}{|c|c|c|c|c|c|}
\hline & MO(Y)D04L2 & MO(Y)D05L2 & MO(Y)D06L2 & MO(Y)D07L2 & MCD12Q1.005 \\
\hline Data Group & Atmosphere & Atmosphere & Atmosphere & Atmosphere & Land \\
\hline Project Type & Swath & Swath & Swath & Swath & Sinusoidal \\
\hline \#Source Data Files & $65 \mathrm{~K}$ & $130 \mathrm{~K}$ & $130 \mathrm{~K}$ & $130 \mathrm{~K}$ & 150 \\
\hline Data Size & $85 \mathrm{~GB}$ & $500 \mathrm{~GB}$ & $2 \mathrm{~TB}$ & $850 \mathrm{~GB}$ & 13GB \\
\hline & MCD15A2.005 & MCD43B3.005 & MO(Y)D11_L2.005 & MO(Y)D13A2.005 & \\
\hline Data Group & Land & Land & Land & Land & \\
\hline Project Type & Sinusoidal & Sinusoidal & Sinusoidal & Swath & Total \\
\hline \#Source Data Files & $7 \mathrm{~K}$ & $7 \mathrm{~K}$ & $113 \mathrm{~K}$ & $3 \mathrm{~K}$ & $585 \mathrm{~K}$ \\
\hline Data Size & $13 \mathrm{~GB}$ & 110GB & $337 \mathrm{~GB}$ & 40GB & $3.9 \mathrm{~TB}$ \\
\hline
\end{tabular}

Even if the above process were manageable and undertaken by scientists, there is still a significant challenge in finding/obtaining the necessary computational capacity to perform the analysis. An entire end-to-end computational run for the US continent over 10 years would require tens of thousands of CPU hours of continuous processing. Clearly, the MODIS data presents unprecedented opportunity for scientific exploration, but there remain significant practical issues that prevent such discoveries, particularly for resource-constrained scientists. 
Therefore, to help these and other scientists, we have designed and implemented a MODIS data reprojection and reduction pipeline in the Windows Azure platform. We choose to leverage cloud computing to provide on-demand computational resources and highly scalable infrastructure for our data processing pipeline based on the observation that the MODIS data files can be processed largely in parallel. Thus we can map the reprojection or scientific computation tasks on these data files to a large number of parallel computational instances in the cloud. Through the implementation of this pipeline, our goals have been to provide the following main contributions for scientists pursuing reprojection/reduction of the MODIS data:

- Reduce or eliminate the issues involved in data collection, data transformation, and data management by constructing a complete data management and task scheduling software framework in Windows Azure. The scientist can make requests to the pipeline using a GUI tool to specify their data requirements, which will then be automatically processed by the pipeline to collect, reproject, integrate the source data, and then perform analytical computation on them. The scientist will receive email notification when the requested task has been finished, and the processing log can be accessed and downloaded for review.

- Leverage cloud computing to provide a highly-flexible and scalable data processing pipeline architecture. In principle, the number of computational instances in the cloud can be adjusted at runtime to support different scales of data processing tasks, thus to accommodate continental or even global scope of MODIS scientific research.

- Scientists can specify a custom reduction step, in which scientists will perform synthesis and analyses computation on the unified data produced after the reprojection step, and will usually reduce these data into a much smaller set of final science data results. We enable our scientists to specify different reduction algorithms in their own tools such as compiled MATLAB application or other command-line tools. They can upload that to the computing instances in Windows Azure using an upload tool.

We believe that this reprojection and reduction of the MODIS data is desired by a number of scientists today who would otherwise be unable to utilize the MODIS data. Furthermore, we believe our architecture can be applied to data other than the MODIS data.

\section{Pipeline Implementation}

\subsection{Pipeline Stage Overview}

Our MODIS data reprojection and reduction pipeline consists of three main stages as shown in Figure 1. Each stage is an independent component and addresses a specific type of data-centric tasks:

Data collection stage: At this stage the data collection model assembles all the required source data according to a specific user request. Each task first determines and then downloads all of the necessary source files for the specified target images. Azure tables are used to hold the metadata describing the FTP site, the remote file structure, and geographical information about the source file. Once the metadata for the specified source files are queried from the meta-tables, it downloads the files from the FTP sites and uploads them into the data repository in Azure blob storage. When all the source data for a specific target are in the data repository, it will initiate a reprojection task for that target by sending a message to the reprojection task queue which will then fetched by the next stage.

Reprojection stage: This stage performs reprojection tasks on the source data collected in 
the first stage. Each task produces one reprojected target image. While the total number and size of data processing requirements are large, the computation requirement for a single task is relatively small. Typically, a single reprojection task requires a collection of only 3-4 source data files, each in the size of several MBs to tens of MBs. The computation required to produce a target sinusoidal file (i.e., project the source data into a uniform format) can be finished in several minutes on a single Azure computing instance.

Analysis and reduction stage: The (optional) reduction stage works on the data produced by the reprojection stage to perform custom reduction analysis algorithms as specified by the scientists. Each task may take any number of input source images and produces a number of reduced image files. Our implementation places some limits on the reduction algorithm that is uploaded by the scientist. First, the reduction tool must be a command line executable and all custom libraries other than the .NET framework must be included with the tool. Second, since the input and output data images are downloaded from/uploaded to the data repository by the Azure instance rather than the reduction tool, the scientist will have to specify the local paths for the input and output images or just use the default local input and output paths. After the reduction algorithm is executed, the output image file will be uploaded to the data repository in Azure blob storage, and a notification email will be sent to the requester. Scientists can then download the results at any time using a download tool.

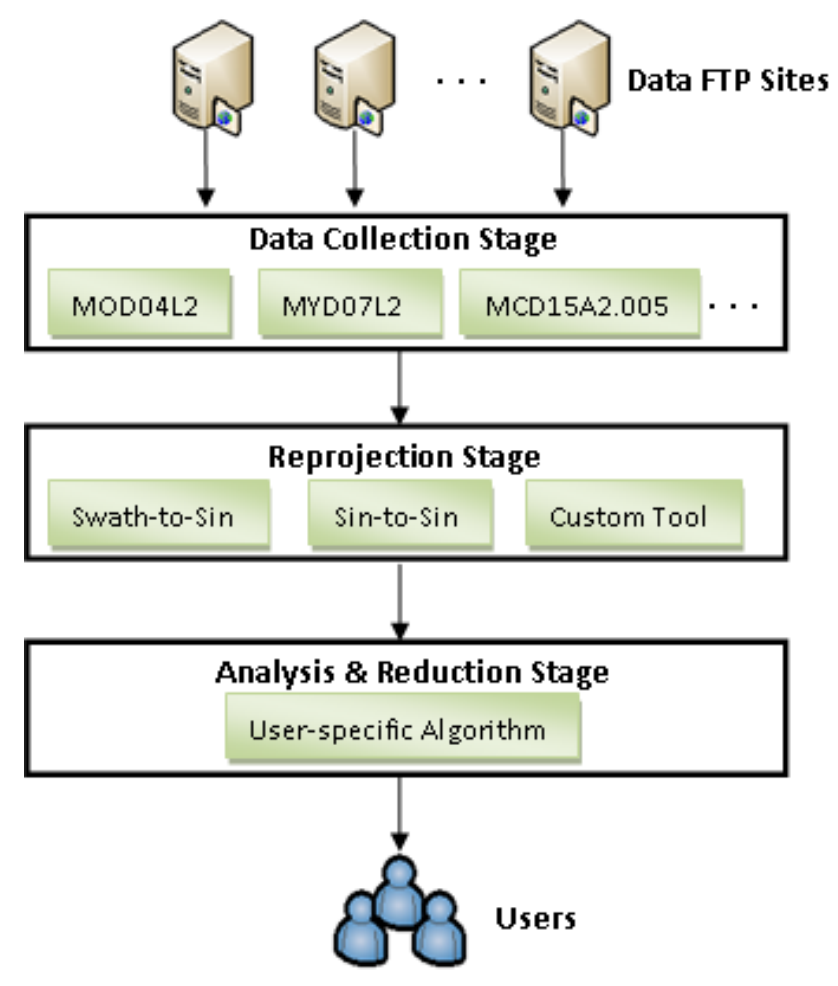

Figure 1. MODIS pipeline implementation in Windows Azure

\subsection{Data Repository}

The data repository serves as a long-term or short-term cache for all sharable data, including the source data, intermediate reprojected data, and final result data. All these data are stored as blobs 
in the Azure storage and grouped in different blob containers. A meta-table that includes the metadata for each single blob is implemented to track the status of the data repository. Whenever a new blob is added or an existing blob is removed, the corresponding metadata entity in the meta-table is updated. The meta-table also keeps record for each blob on which user request/task it is connected to. By keeping track of such information we can automatically manage the data produced by a large group of users and enable the storage resource sharing between different users. Users don't need to worry about the details such as semantic groupings and locations of their data. Using a GUI data management tool, they can simply retrieve and manage their data using their request IDs.

An important design aspect that has not been incorporated into the implementation to date is security with regard to intermediate data products, final data products, and uploaded reduction algorithms. We plan to implement fine-grained access control for this important data in a future version of the pipeline by leveraging the .NET Access Control Service [19] in the Windows Azure platform.

\subsection{Task Scheduling}

Each stage in the pipeline has a task manager worker role and a number of computational worker roles. The task manager fetches user requests from the request queue; parses the user request into a large number of parallel data collection, reprojection, and reduction tasks (if specified); and then dispatches the large number of tasks into the task queues. During the processing of a user request in the pipeline, the task scheduler also keeps track of the task statuses and performs several fault tolerance actions. Each computational worker role instance fetches tasks from the task queue and performs the corresponding data processing work.

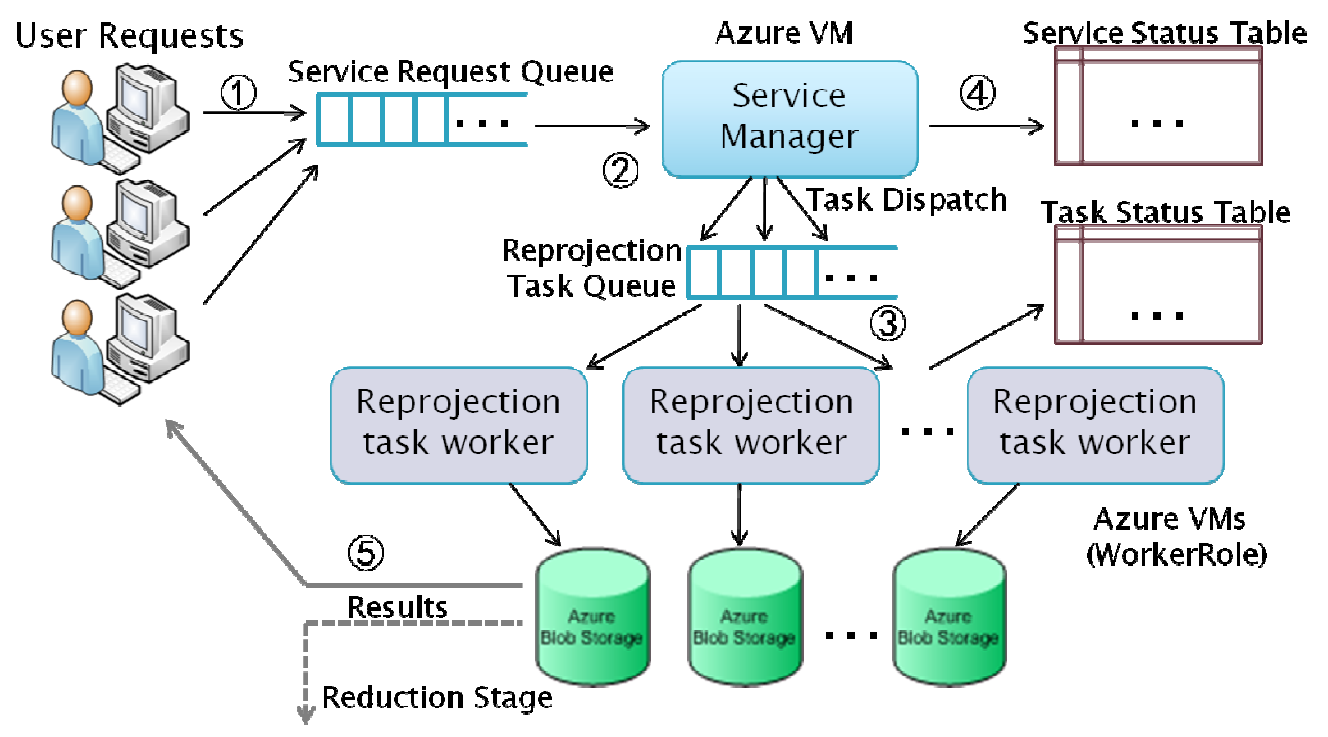

Figure 2. Reprojection task scheduling.

Figure 2 shows the implementation detail of the reprojection stage. A typical reprojection computation follows the following steps:

1. The user sends a reprojection request to the service request queue using a desktop client tool.

2. The reprojection task service manager checks the service request queue for the arrival of a new request. Upon arrival, the user request is fetched and parsed into a number of parallel 
data collection and reprojection tasks. The reprojection task manager then dispatches the tasks into the collection and reprojection task queues. It then registers the metadata of this new reprojection service in the service status table, and keeps monitoring the status of the reprojection service.

3. Each of the reprojection computational worker role instances keeps fetching and executing the reprojection tasks from the task queue. When the worker gets a task from the queue, it first parses the specification for that single reprojection task, queries a number of meta-tables for the corresponding source data items collected in stage 1, and then downloads those data images from the blob storage into temporary local files. It then reprojects the source data files into intermediate data image results in the format as specified by the user request. When the reprojection is done, it uploads the result back to a specified blob storage location, and then updates the information for that task in the task status meta-table.

4. The service monitor keeps checking the task status table to see if all single tasks for a user request have been finished. When it's finished, it sends a notification email back to the user.

5. When the user receives the notification email, he/she can either download the reprojected data to the local machine using a download tool, or specify another reduction request to do analytical computation on that set of data. Note, however, that although here the scientist uses two separate requests for the reprojection and reduction tasks, in practice he/she can combine them into a single request and thus will not be interfered by the intermediate reprojected results notification step.

The task scheduling for the data collection and reduction stages are similar to the reprojection stage, and we will not introduce their implementation details here.

\subsection{Fault Tolerance}

Besides task scheduling and service monitoring, the different task manager worker roles also perform a set of fault tolerance actions since tasks can fail due to a lot of reasons. The service manager keeps the track of each single task that are under processing and if a task doesn't finish after an unusual amount of time, it will send the same task to the task queue again which can be restarted by another task worker role. However, if that task still fails after totally 3 retries, it will be most likely that the task failure is caused by some external errors such as crashed source data files. Then the service manager will give up the retry and mark that task as failed. Note that, however, users are usually requesting for a large amount of tasks and doing analysis on all of the result data, and a single failed task doesn't necessarily mean the whole user request has totally failed. In order to diagnose the various system failure problems and help scientist monitor the progress of their requests, we also developed a tool to monitor the statuses of all the single tasks that a user has requested. The tools can show for each task whether it is staying in the queue, under processing in a worker role instance, or has been finished.

\section{Evaluation}

We tested our pipeline implementation in Windows Azure using an account that can acquire a maximum of 200 virtual machine instances. Since the reprojection stage is the most computeand data-intensive stage, and it also involves the most frequent usage of all 3 types of Azure storage services (blob, queue, and table service), thus we choose the reprojection task processing for our performance and scalability test. 


\subsection{Single instance performance}

We first compare the performance of a single computational instance in Azure with that of a high-end desktop machine. Table 2 shows the capacity of the desktop machine we used for the test and the approximate specification of an Azure instance. We did our experiment by executing totally 45 different reprojection tasks on both the desktop machine and a single Azure instance. Each reprojection task involves the following operations: 1 Azure table query to get the list of source data files that are required for the given reprojection task; 4 8 table queries for the metadata of each source data file; downloading all source data files from Azure blob storage to local storage; performing reprojection computation on downloaded source data and generating the reprojected data.

Table 2. Capacity of desktop machine and a single Azure instance

\begin{tabular}{|l|l|l|}
\hline & \multicolumn{1}{|c|}{ Desktop } & \multicolumn{1}{|c|}{ Azure Instance } \\
\hline \multirow{4}{*}{ Capacity } & CPU: Intel Core2Duo E6850 @ 3.0GHZ & CPU: 1.5-1.7GHZ X64 equivalent processor \\
& Memory: 4GB & Memory: 2GB \\
& Hard Disk: 1TB SATA & Local Storage: 250GB \\
& Network: 1Gbps Ethernet & Network: 100Mbps \\
& OS: Windows 7 RC Build7100 (32-bit) & OS: Windows 2008 Server x64 (64-bit) \\
\hline
\end{tabular}

In our experience, the time required for the reprojection tasks of different products can differ widely due to different characteristics of the source data. Thus we selected the 45 reprojection tasks from 3 different data products (15 tasks for each): MOD04L2, MOD06L2, and MYD11L2.005, to show the variability across different data product characteristics. For each task, we measured its wall-clock time, i.e. the period from when the worker role instance or the desktop machine starts processing the task to the time when it finishes the reprojection computation. We then separated the total wall-clock time into two parts of measurements:

- The Azure Storage Service communication time, which is used to query metadata from Azure tables, download source data files from Azure blob storage, etc.

- The local reprojection time, which is used to perform reprojection computation on downloaded source data files.

Table 3. Execution statistics on desktop and a single Azure instance

a) MOD04L2

\begin{tabular}{|c|c|c|c|}
\hline & Wall Clock Time(avg.) & Comm. Time(avg.) & Reprojection Time(avg.) \\
\hline Desktop & $39.1 \mathrm{~s}$ & $9.2 \mathrm{~s}$ & $29.9 \mathrm{~s}$ \\
\hline Azure Instance & $82.7 \mathrm{~s}$ & $14.9 \mathrm{~s}$ & $67.7 \mathrm{~s}$ \\
\hline Perf. Ratio & 2.1 & 1.6 & 2.3 \\
\hline o(Perf. Ratio) & 0.21 & 0.79 & 0.13 \\
\hline
\end{tabular}

b) MOD06L2

\begin{tabular}{|c|c|c|c|}
\hline & Wall Clock Time(avg.) & Comm. Time(avg.) & Reprojection Time(avg.) \\
\hline Desktop & $169.5 \mathrm{~s}$ & $65.8 \mathrm{~s}$ & $103.7 \mathrm{~s}$ \\
\hline Azure Instance & $267.5 \mathrm{~s}$ & $52.6 \mathrm{~s}$ & $214.9 \mathrm{~s}$ \\
\hline Perf. Ratio & 1.6 & 0.80 & 2.07 \\
\hline o(Perf. Ratio) & 0.13 & 0.31 & 0.07 \\
\hline
\end{tabular}


c) MOD11L2.005

\begin{tabular}{|c|c|c|c|}
\hline & Wall Clock Time(avg.) & Comm. Time(avg.) & Reprojection Time(avg.) \\
\hline Desktop & $80.3 \mathrm{~s}$ & $14.9 \mathrm{~s}$ & $65.4 \mathrm{~s}$ \\
\hline Azure Instance & $151.5 \mathrm{~s}$ & $15.3 \mathrm{~s}$ & $136.1 \mathrm{~s}$ \\
\hline Perf. Ratio & 1.9 & 1.0 & 2.1 \\
\hline o(Perf. Ratio) & 0.39 & 0.73 & 0.40 \\
\hline
\end{tabular}

Table 3(a)-(c) shows the statistics on the execution of all 45 tasks on both the desktop machine and the Azure instance. Each table shows statistics for the 15 reprojection tasks from each data product. The Wall Clock Time shows the average end-to-end time (in seconds) for a single task across all 15 tasks. Similarly, the Comm. Time and Reprojection Time show the average network communication time and reprojection computation time across all 15 tasks (both in seconds). The columns show the comparison of the results between the desktop machine and the Azure instance. The Perf. Ratio shows the performance ratio of the two and is calculated by Azure Instance Time / Desktop Time. Specifically, $\sigma($ Perf. Ratio) shows the standard deviation of the performance ratio across all 15 tasks. As we can see from above tables, due to the large capacity gap between the single Azure instance and the high-end desktop machine, in all cases the wall clock time for each task on Azure instance is larger than that on the desktop by a factor of 1.6 2.1. This is not surprising because according to the Azure instance capacity specification, its hardware capacity is not as good as that of our desktop machine.

As for the separated measurements, the statistics on network communication time illustrate some interesting behaviors. First, the Azure storage communication performance ratios of Azure instance and desktop machine are not consistent across all 3 data products. Second, the standard deviation of communication performance ratio is very large and can account for as much as 70\% of the performance ratio itself. To further demonstrate this behavior in detail, Table 4 shows a detailed list of communication time measurements for all 15 tasks in MYD11L2.005 product, which has the largest standard deviation in total percents of the performance ratio. As we can see, the communication performance ratios for each of the 15 tasks present a random characteristic. This indicates that the communication time required for a task is mostly affected by the highly inconsistent response time of the Azure Storage Services, rather than the Internet connection bandwidth. Furthermore, although the Azure instances are running in the same datacenter as the storage location of our source data (Users can select which datacenter to be the storage location for their data), it seems these instances didn't share any benefits from its internal network connection advantages because its performance is not even as good as a machine connected from outside the datacenter.

Table 4. Communication Time for all 15 tasks in MYD11L2.005 (Unit: seconds)

\begin{tabular}{|c|c|c|c|c|c|c|c|c|c|c|c|c|c|c|c|}
\hline Task & $\mathbf{1}$ & $\mathbf{2}$ & $\mathbf{3}$ & $\mathbf{4}$ & $\mathbf{5}$ & $\mathbf{6}$ & $\mathbf{7}$ & $\mathbf{8}$ & $\mathbf{9}$ & $\mathbf{1 0}$ & $\mathbf{1 1}$ & $\mathbf{1 2}$ & $\mathbf{1 3}$ & $\mathbf{1 4}$ & $\mathbf{1 5}$ \\
\hline Desktop & 12 & 16 & 14 & 18 & 19 & 9 & 21 & 16 & 7 & 27 & 14 & 6 & 23 & 6 & 16 \\
\hline Azure Instance & 7 & 18 & 17 & 18 & 17 & 16 & 16 & 19 & 16 & 20 & 19 & 5 & 18 & 19 & 5 \\
\hline Perf. Ratio & 0.58 & 1.13 & 1.21 & 1.00 & 0.89 & 1.78 & 0.76 & 1.19 & 2.29 & 0.74 & 1.36 & 0.83 & 0.78 & 3.17 & 0.31 \\
\hline
\end{tabular}

On the other hand, the statistics on the reprojection computation time indicate a much better consistency, which indicates the abstraction of the hardware resource capacity as provided by Azure instances are much more stable in performance. 


\subsection{Pipeline Performance and Scalability}

One of the main advantages of cloud computing is high flexibility and scalability to provide ondemand computing resources. Our pipeline architecture is very flexible in terms of the ability to dynamically adjust to the scale of computational requirements by the scientific data processing tasks. To see how the performance of our pipeline scales with the number of the computing instances, in this experiment we measured the total processing time for running 1500 reprojection tasks from each of the above 3 data products using different number of Azure instances. For each data product, we run the same 1500 tasks using 150, 100, and 50 Azure instances, and measure the total processing time for all tasks to be finished. We then estimated the speedups we get when comparing to the capacity of a single Azure instance and that of our high-end desktop machine. Table 5 shows the total processing time (in hours) for each run. Azure pipeline runs with 150, 100, and 50 instances are all measured from real tests, while the single Azure instance and desktop processing time is only an estimation, since it would take days to finish the whole run. To calculate the single Azure instance and desktop processing time for the 1500 tasks for each data product, we first get the average wall clock time for a single task as we measured in Section 6.1, and then multiply it by 1500 .

Table 5. Total processing time for 1500 reprojection tasks (Unit: hours)

\begin{tabular}{|c|c|c|c|}
\hline & MOD04_L2 & MOD06_L2 & MYD11_L2.005 \\
\hline 150 instances & 0.30 & 0.85 & 0.44 \\
\hline 100 instances & 0.40 & 1.20 & 0.61 \\
\hline 50 instances & 0.76 & 2.25 & 1.12 \\
\hline Single instance & 34.21 & 116.19 & 63.56 \\
\hline Desktop & 16.29 & 72.62 & 33.45 \\
\hline
\end{tabular}

From the table above we can calculate the speedups for running multiple Azure instances over a single instance or a single desktop machine, and the results are shown in Figure 3. The "VMs" in the figure indicates the Azure virtual machine instances. As the figure shows, the performance of our pipeline scales almost lineally with the number of instances, and although each Azure instances has a moderate capacity as compared to our desktop machine, in best case we still get a nearly 90x speedup over a single desktop when running tasks for MOD06L2 on 150 Azure instances. As compared to the performance of a single Azure instance, we almost get the same times of speedups as the number of instances in all cases except for product MOD04_L2.

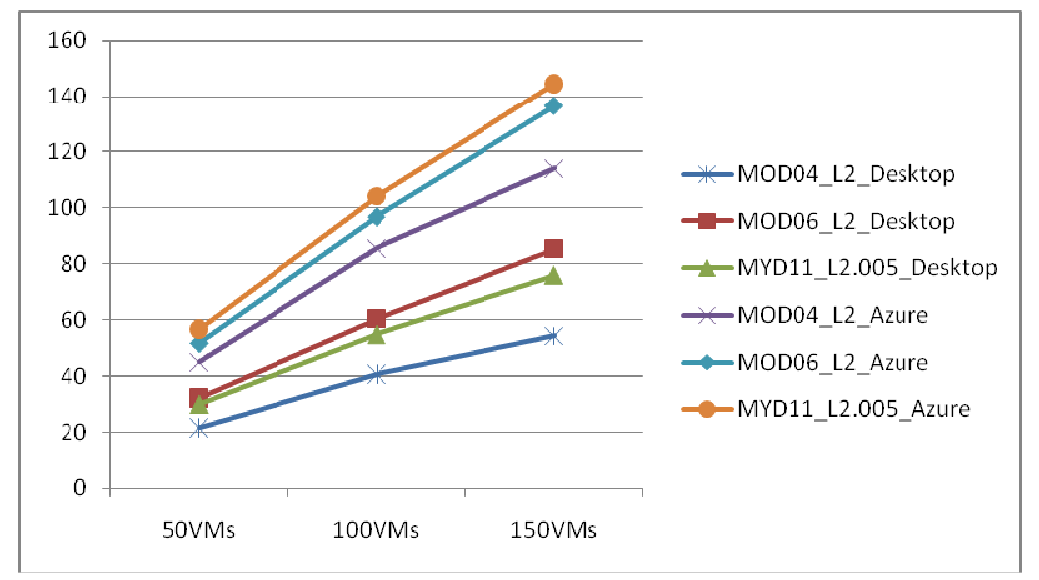

Figure 3. Pipeline performance speedups over a single desktop machine 


\subsection{Scalability of the Azure Storage Service}

Since there are many Azure instances running concurrently in the pipeline, they will frequently perform concurrent operations such as table queries, queue/dequeue operations, blob uploads/downloads, etc. thus we expect that the number of concurrent working instances in Azure will impact the response performance of Azure Storage Services. To measure how well the Azure Storage Services scale with the number of concurrent instance requests, for each of the reprojection runs on product MOD06L2 in Section 6.2, we also measured the total Azure storage service communication time and the computation time, i.e. we sum up both the communication time and the reprojection time for each of the 1500 tasks. We choose product MOD06L2 for our measurements because reprojection tasks for this product requires downloading the largest size of source data ( 100MB for each task), and thus can present the severest concurrent operation request behaviors for Azure Storage Services.

Figure 4 shows the total reprojection time and communication time for all 1500 tasks for MOD06L2 product. When only 50 Azure instances are running concurrently, the total Azure Storage Service communication time is a little less than 20 hours, while it becomes more than 27 hours when 150 instances are running concurrently. This result indicates that the scalability is not so free when it comes to the Azure cloud storage service and the latency can get significantly larger when there are many concurrent, long-last requests (such as blob downloads) to the same queue, table, and blob storage structure.

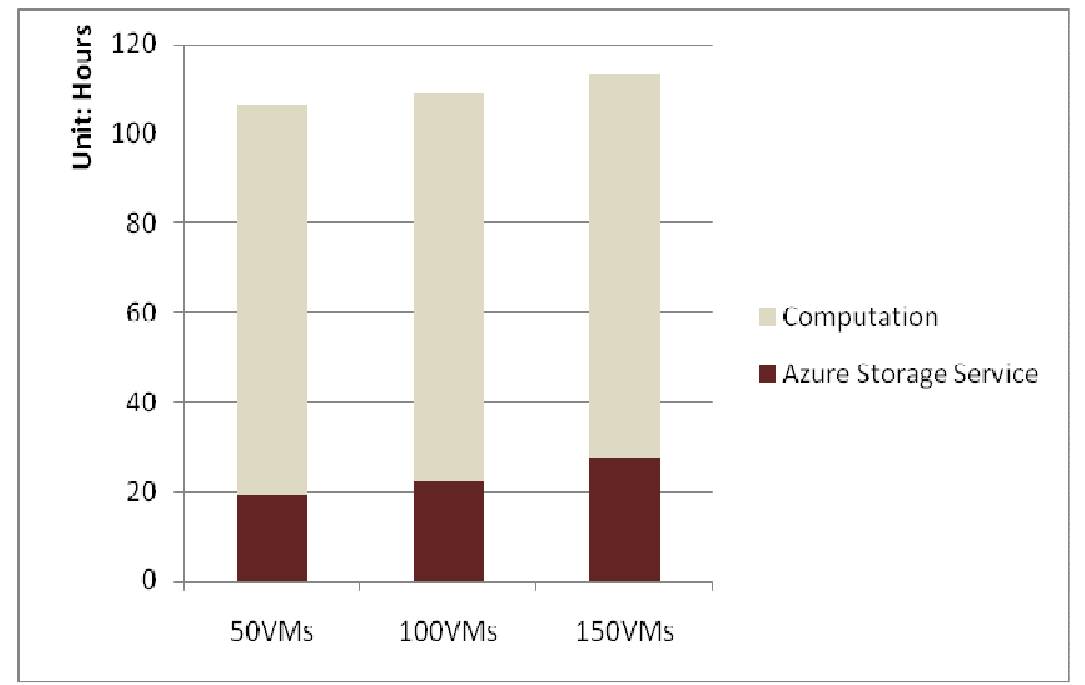

Figure 4. Reprojection time VS. Communication time for MOD06L2 product

\section{Conclusion}

In this paper, we have described how we have leveraged cloud computing to create a MODIS satellite data reprojection and reduction pipeline. We are currently undergoing user studies involving biometeorologists, in part to validate the results from our first processing of a single year covering the whole US continent. We expect to launch the remaining 10 year computation for the US shortly and look forward to global scale. With the flexibility and scalability provided by the cloud computing model, scientists who previously only worked on their desktop machines can now access powerful computing resources and to scale their research scopes in a costeffective way. Our implementation of the MODIS data processing pipeline in Windows Azure 
has further demonstrated the potential of using cloud computing to lower a series of data entry barriers for eScience by leveraging the scalable storage services.

Through the design and implementation of this MODIS application, we have first-hand experience with a number of issues for the broader escience community. Commercial cloud computing infrastructures today are, to a large extent, running behind the scene. This infrastructure transparence makes it more difficult for cloud application developers to diagnose problems as they occur. In our experience, troubleshooting problems in cloud applications is not only time-consuming, but sometimes can also be very tricky. As a result, managing code dependencies took a noticeable part of our development time. Local simulation and debugging for cloud applications is another remaining challenge. While Windows Azure provides a local simulation environment for cloud application development, there are many cases that applications will run into different behaviors when the application is deployed, which is due to the heterogeneity of the underlying software and hardware environments where the cloud applications are actual running. Therefore, we believe the transparency of the underlying cloud infrastructure is currently a general challenge for cloud application developers and has inevitably raised the application development and deployment barrier.

A notable aspect of our current pipeline is that it does not leverage an existing MapReduce framework such as Hadoop [20] or Dryad [21]. There are two reasons for this. First, none of these frameworks are currently supported in Windows Azure Platform. Windows Azure is still in Community Technology Preview phase, and it is expected that more frameworks such as Dryad will be supported in near future. Second, our scientists' data flow and management pattern are similar to, but not exactly the same as MapReduce. Our reprojection stage (which is the Map) and the reduction stage (which is the Reduce) are more loosely coupled and the results from the reprojection stage can be reused across multiple reductions. Nevertheless, we are still interested to see how our pipeline architecture could be implemented on an existing MapReduce framework. Thus, it remains to be our future work.

In conclusion, as we have demonstrated in our work, cloud computing has the great potential to eliminate or significantly lower many resource barriers and data barriers that have been existing for a long time in eScience domain. It provides a cost-effective, highly scalable and flexible solution for those large-scale, compute- and data-intensive eScience researches. At the same time, at this early stage of cloud computing, some general challenges remain to be overcome and tradeoffs need to be made by scientists and developers when consider moving their applications to the cloud.

\section{References}

[1] NASA MODIS Web page. http://modis.gsfc.nasa.gov/

[2] C. Justice et al., "The Moderate Resolution Imaging Spectroradiometer (MODIS): land Remote Sensing for Global Change Research," IEEE Transactions on Geoscience and Remote Sensing, 36(4): 1313-1323, 1998.

[3] Huete, A., Didan, K., Miura, T., Rodriguez, E. P., Gao, X., \& Ferreira, L. G. (2002). Overview of the radiometric and biophysical performance of the MODIS vegetation indices. Remote Sensing of Environment, 83, 195- 213

[4] W. E. Esaias, M. R. Abbott, I. Barton, O. B. Brown, J.W. Campbell, K. L. Carder,D.K. Clark, R. H. Evans, F. E. Hoge, H. R. Gordon,W. M. Balch, R. Letelier, and P. J. Minnett, “An 
overview of MODIS capabilities for ocean science observations," IEEE Trans. Geosci.

Remote Sensing, vol. 36, pp. 1250-1265, July 1998.

[5] Microsoft's Windows Azure Platform. http://www.microsoft.com/azure/default.mspx

[6] Amazon Elastic Compute Cloud (EC2). http://aws.amazon.com/ec2/

[7] Google App Engine. http://code.google.com/appengine/

[8] Constantinos Evangelinos and Chris N. Hill, "Cloud Computing for Parallel Scientific HPC Applications: Feasbility of Running Coupled Atmosphere-Ocean Climate Models on Amazon's EC2", 2008, CCA-08.

[9] P. Watson, P. Lord, F. Gibson, P. Periorellis, and G. Pitsilis, "Cloud Computing for eScience with CARMEN," Iberian Grid Infrastructure Conference, May 2008.

[10] K. Keahey, R. Figueiredo, J. Fortes, T. Freeman, and M. Tsugawa, "Science Clouds: Early Experiences in Cloud Computing for Scientific Applications," Cloud Computing and Applications, 2008.

[11] C. Hoffa, G. Mehta, T. Freeman, E. Deelman et al., "On the Use of Cloud Computing for Scientific Workflows", IEEE Fourth International Conference on eScience (eScience 2008), Dec. 2008.

[12] E. Deelman, G. Singh, M. Livny, B. Berriman, J. Good. "The Cost of Doing Science on the Cloud: The Montage Example," Proceeding of Super Computing 2008, November 2008.

[13] C. Vecchiola, M. Abedini, M. Kirley, X. Chu, and R. Buyya, "Classification of Gene Expression Data on Public Clouds," Technical Report, CLOUDS-TR-2009-7, Cloud Computing and Distributed Systems Laboratory, The University of Melbourne, Australia, Aug. 7, 2009.

[14] Z. Hill and M. Humphrey. A Quantitative Analysis of High Performance Computing with Amazon's EC2 Infrastructure. In Proceedings of the $10^{\text {th }}$ IEEE/ACM International Conference on Grid Computing (Grid 2009). Oct 13-15 2009. Banff, Alberta, Canada

[15] Christian Baun, Marcel Kunze Performance measurement of a private Cloud in the OpenCirrus Testbed. 4th Workshop on Virtualization in High-Performance Cloud Computing (VHPC 2009). Aug 2009.

[16] "Primary Data Search," https://wist.echo.nasa.gov/api/

[17] MODIS Swath Reprojection Tool.

http://gcmd.nasa.gov/records/MODIS_Swath_Reprojection_Tool.html

[18] Msphinx. http://www-loa.univ-lille1.fr/Msphinx/

[19] .NET Access Control Service. http://www.microsoft.com/azure/accesscontrol.mspx

[20] "Hadoop MapReduce Framework," http://hadoop.apache.org/mapreduce/.

[21] M. Isard, M. Budiu, Y. Yu, A. Birrell, and D. Fetterly, "Dryad: Distributed data-parallel programs from sequential building blocks,” In EuroSys 2007, March 2007. 